\title{
Philosophiques
}

\section{Universalisme et pluralisme chez Montesquieu}

\section{Philip Knee}

Volume 18, numéro 2, automne 1991

URI : https://id.erudit.org/iderudit/027150ar

DOI : https://doi.org/10.7202/027150ar

Aller au sommaire du numéro

Éditeur(s)

Société de philosophie du Québec

ISSN

0316-2923 (imprimé)

1492-1391 (numérique)

Découvrir la revue

Citer cet article

Knee, P. (1991). Universalisme et pluralisme chez Montesquieu. Philosophiques, 18(2), 3-26. https://doi.org/10.7202/027150ar

\section{Résumé de l'article}

Ce texte propose une lecture de l'oeuvre de Montesquieu à partir de débats récents sur le relativisme politique. La méthode d'ensemble qui se dégage de quelques grands thèmes des Lettres persanes et de L'Esprit des lois permet d'apercevoir le double enseignement de cette pensée: qu'elle est structurée à la fois par un horizon normatif d'universalité et par une conception plurielle du bien politique. d'utilisation que vous pouvez consulter en ligne.

https://apropos.erudit.org/fr/usagers/politique-dutilisation/ 


\title{
ARTICLES
}

\section{UNIVERSALISME ET PLURALISME CHEZ MONTESQUIEU*}

\author{
par Philip Knee
}

\begin{abstract}
RÉSUMÉ. Ce texte propose une lecture de l'œuvre de Montesquieu à partir de débats récents sur le relativisme politique. La méthode d'ensemble qui se dégage de quelques grands thèmes des Lettres persanes et de L'Esprit des lois permet d'apercevoir le double enseignement de cette pensée: qu'elle est structurée à la fois par un horizon normatif d'universalité et par une conception plurielle du bien politique.

ABSTRACT. This paper proposes a reading of Montesquieu's work on the basis of recent debates concerning political relativism. The overall method which is present in some of the major themes of the Lettres persanes and De l'Esprit des lois suggests that this thought is structured at once by a normative horizon of universality and by a plural conception of the political good.
\end{abstract}

Il est courant à notre époque d'identifier le maintien d'un étalon universel en matière de jugement moral ou politique à un impérialisme culturel, à la volonté d'une culture particulière $\mathrm{d}^{\prime}$ assimiler ce qui est différent d'elle. On dénonce alors ce discours réducteur dont l'énonciateur, par l'univers absolu et unique qu'il s'est ainsi créé, est devenu incapable de se voir, car se voir implique de passer par l'autre, et cet autre, une fois réduit, ne s'appartient plus: il ne dit plus rien que ce que dit de lui le discours universaliste. Mais c'est ici que les problèmes commencent, car cette critique fort répandue de l'universalisme hypocrite de l'Occident peut mener au repli de chacun sur sa seule différence. La revendication d'un « droit

* Ce texte s'inscrit dans un projet de recherche sur la pensée française aux $18 \mathrm{e}$ et $19 \mathrm{e}$ siècles, soutenu par le Conseil de recherches en sciences humaines du Canada. 
à la différence" peut signifier que chaque culture, chaque nation ou groupe, aurait son principe d'évaluation en soi, ce qui fait disparaître, au nom de la diversité, tout étalon universel pour juger les conduites. Dès lors les adversaires dans un conflit ne sont plus que des forces qui s'opposent, ils ne sont plus des semblables pouvant trouver, ou au moins chercher, les termes communs d'un dialogue en s'appuyant sur une universalité de justice ou de droit.

Historiquement, c'est au tournant du XVIIIe au XIXe siècle qu'on situe souvent la cristallisation de ces enjeux. Dès l'époque de la Révolution française, les idéaux triomphants de la pensée des Lumières sont mis en cause; par Burke, par exemple, qui condamne l'abstraction de ces idéaux au nom des coutumes, des traditions, des bons "préjugés" de chaque nation. Peu après, les conquêtes de Napoléon, menées au nom des idéaux révolutionnaires, sont perçues comme incarnant une volonté d'assimilation culturelle, et c'est avec sa défaite qu'on voit surgir, dans le romantisme en Allemagne ou le nationalisme en France, une glorification de la particularité des cultures. L'opposition qui ainsi se fait jour met aux prises une légitimité de droit, fondée sur une idée universelle de l'homme, sur son autonomie, contre les préjugés qui le maintiennent dans l'ignorance; et une légitimité de fait, fondée sur l'enracinement, sur l'esprit d'un peuple, sa langue ou son passé. Cette opposition aide à comprendre nos débats sur la culture au XXe siècle; mais ils sont difficiles à déchiffrer dans la mesure où les armes de cette tradition d'appartenance servent à combattre l'impérialisme culturel (en particulier le colonialisme) paradoxalement au nom même de l'idéal des Lumières. En effet, l'idéal de la culture comme compréhension des différences, que la pensée des Lumières opposait au fanatisme et à la superstition au XVIIIe siècle, s'est perverti, car la culture n'est souvent définie aujourd'hui que comme une appartenance ou une origine. Elle peut alors servir à justifier, au nom des droits et des différences, tous les fanatismes politiques ou religieux, ces fanatismes qu'il s'agissait justement de combattre au départ. Et c'est de ce retournement dans la pensée que s'alimentent au XXe siècle beaucoup de causes nationales, tiers-mondistes en particulier. Même quand on prétend s'en réclamer, l'idéal des Lumières se tourne en son contraire lorsque, au nom du différent, on en vient à nier la communauté de conscience entre les hommes et tout horizon d'universalité. En revanche, dira-t-on, ce danger n'a-t-il pas son envers? L'affirmation d'une 
telle universalité n'ouvre-t-elle pas inévitablement la voie à un modèle unique de société, à une pratique politique réductrice directement régie par les normes de perfection ou d'excellence dictées par un savoir philosophique?

Depuis quelques années plusieurs auteurs ont voulu prendre en charge ces interrogations en se penchant à nouveau sur la philosophie des Lumières ${ }^{1}$, afin d'y chercher l'expression d'une universalité morale et politique permettant d'éviter certains écueils de la pensée contemporaine: celui d'un improbable " retour » à la tradition ancienne du droit naturel comme seule issue pour affronter le relativisme et l'historicisme modernes; celui d'un anti-humanisme (fort répandu, surtout en France, depuis une trentaine d'années) découlant du détournement scientiste et positiviste des Lumières au XIXe siècle ${ }^{2}$. Dans ce contexte on peut s'interroger sur le statut accordé à Montesquieu par certains de ces auteurs ${ }^{3}$. Alors qu'une réputation tenace continue à faire de lui seulement le précurseur de la sociologie positiviste, ils suggèrent que sa perspective d'un «droit naturel rationalisé " renferme bien autre chose qu'une simple science objective des faits et des lois. Il s'agira ici de revenir sur cette pensée, non pour reformuler comme telles ses thèses bien connues sur la séparation des pouvoirs, la nature des gouvernements ou l'influence des climats; mais pour dégager brièvement, à partir de ces thèmes, deux traits significatifs de sa philosophie, qui permettent peut-être de mieux apercevoir son actualité et sa pertinence dans les débats actuels.

On peut dire que cette philosophie est structurée de bout en bout par une ambivalence: celle de l'universel du droit politique et du particulier de l'esprit des peuples; ambivalence dont l'idée de loi dans De L'Esprit des lois est la médiation et dont l'enjeu épistémologique est présent dès les Lettres persanes. C'est cette ambivalence que nous chercherons à interroger afin de mettre en évidence, à la fois, que cette pensée est structurée par un horizon normatif d'universalité, et qu'elle est animée d'une conception plurielle du bien politique. Pour cela nous caractériserons d'abord la méthode mise en oeuvre par Montesquieu dans ses deux principaux ouvrages ${ }^{4}$, où le thème de la religion nous servira d'entrée en matière; puis nous tenterons de formuler ce double enseignement, universaliste et pluraliste, de sa pensée. 
"Vérité dans un temps, erreur dans un autre", dit Usbek dans les Lettres persanes (LXXV), faisant écho à Montaigne et Pascal, et résumant la vaste entreprise de relativisation des certitudes qui semble être celle de l'ouvrage. En effet, ces Persans en ballade semblent ne s'ouvrir qu'un abîme d'incertitude, car ils ne font pas qu'interroger les moeurs parisiennes (vie de salons, savoirs superficiels, intrigues politiques), mais mettent en cause en même temps leurs propres croyances les plus intimes ( $L P, \mathrm{XVII}$ ). La leçon de Montesquieu est-elle alors qu'on est condamné au relativisme des sens, à la multiplicité des opinions, et à l'impuissance de l'esprit à aller au-delà d'un perpétuel dévoilement de la contingence des êtres? Et que si l'on peut bien être Persan, on ne peut pas être homme? Dans lettre après lettre sur la religion, c'est le caractère changeant, illusoire ou hypocrite des croyances qui est moqué, celles-ci étant renvoyées, par exemple, à la constitution du corps humain: l'immortalité serait crue selon l'influence des désirs terrestres, selon les impulsions physiologiques et psychologiques des hommes. De plus, les idées religieuses semblent calquées sur ce que l'homme imagine sur lui-même, et Dieu se trouve ainsi chargé de toutes les imperfections humaines (LXIX). En religion comme en toutes choses, on juge "par un retour secret que nous faisons sur nous-mêmes " (LIX), et ce retour sur soi se manifeste par les illusions de grandeur contenues dans la religion, alors que les hommes rampent sur un atome: la Terre, qui n'est qu'un point de l'univers (LIX); et ces hommes s'y donnent une importance indue, exprimée par exemple par leurs arguments contre le suicide (LXXVI). D'où une interrogation sur tous les dogmes: la Création, la chute, le péché (CXIII); et une mise en question de toutes les superstitions, surtout de l'esprit missionnaire et des dévots (LXXV et CXLIII); et cela autant pour la religion chrétienne (sur laquelle Usbek et Rica portent le même regard extérieur que les Européens ont sur la Perse) que pour la religion mahométane.

Mais pas plus sur cette question que sur les autres, on va le voir, les Lettres ne débouchent sur un scepticisme désespéré. Cet abîme d'incertitude n'est présent que comme un moment salutaire, un moment d'étonnement philosophique qui est d'emblée dirigé vers autre chose. On n'a pas affaire ici à une entreprise seulement critique, à une infinie démystification des illusions de la vie sociale sans la reconstruction d'un autre monde, car 
ce constat de la fragilité des croyances et de la multiplicité des dogmes et des sectes, induit insensiblement, au fil des échanges, une inversion de perspective: une invitation à considérer la fonction sociale des religions. Le problème de la vérité du dogme devient celui de son utilité, et le questionnement des certitudes se transforme en une méditation sur les formes multiples de la religion et en une invitation à la tolérance. Plus que sur la vérité des croyances, l'attention se porte sur leurs excès, les affrontements auxquels elles conduisent quand elles s'accompagnent de mesures intolérantes ou inutiles ${ }^{5}$. Non que les oppositions religieuses soient en elles-mêmes nocives dans la société; elles peuvent au contraire être utiles (LXXXV); mais seulement dans le cadre d'un esprit de tolérance dont le travail d'observation et de compréhension ici suggère justement la voie. Sans doute trouve-t-on dans les Lettres persanes ce qui, chez Voltaire, est dominant: un travail de sape, une volonté d'ébranler les consciences. Mais cette démonstration (menée d'ailleurs avec une légèreté qui ne tombe jamais dans le sarcasme ou le dénigrement voltairiens) ne fait que préparer l'essentiel: la reconnaissance d'une universalité nouvelle qui peut rendre compte de la diversité et de la constance des religions. Cette universalité est un principe fondamental de la nature humaine, un sentiment intérieur qui anime le coeur de tous les hommes, et qui est discernable à travers la comparaison des pratiques humaines, aussi diverses soient-elles. Au fil des Lettres se dégage l'idée d'un rapport équilibré au monde où la raison dissipe les fausses peurs de l'imagination et reconnait ses propres limites, ces peurs venant justement du refus d'affronter certains phénomènes par la raison et du refus orgueilleux de reconnaitre la relativité de la place de l'homme dans l'univers. On peut sans doute parler d'une religion naturelle ici, car à travers Usbek, Montesquieu suggère clairement que seul importe finalement un amour de la justice qui définit le noyau commun des religions (XLVI). Quel que soit le fondement religieux qu'on leur donne, ces valeurs communes manifestent l'équivalence des différentes religions et un horizon éthique commun à tous les hommes.

Il est vrai que Montesquieu, à la fois par prudence politique et par sa disposition personnelle, ne s'en tient pas à cette affirmation d'équivalence. En passant des Lettres persanes à L'Esprit des lois, il dit d'emblée sa préférence pour la religion chrétienne, et suggère qu'elle incarne cette éthique de la tolérance et qu'elle favorise le bonheur des hommes. Sans doute, comme 
toutes les religions, le christianisme est utile du point de vue politique; et, pour Montesquieu, il se prête particulièrement bien à la stabilisation des sociétés: en adoucissant les moeurs, en souhaitant que les chrétiens remplissent leurs devoirs sociaux et qu'ils aient les meilleures lois politiques et civiles ( $E L, X X I V$, $1)^{6}$. Mais en plus du bien du citoyen, le christianisme fait le bonheur de l'homme, son bonheur dans cette vie en plus de l'autre vie. Aussi indispensable à l'individu qu'à la société, Montesquieu cherchera donc, non pas à «faire croire» sa religion, son point de vue n'étant pas celui du théologien, mais à la " faire aimer " en montrant ses bienfaits, c'est-à-dire en montrant en quoi elle rejoint mieux l'universalité morale que les autres religions. En ce sens, dans l'économie de L'Esprit des lois, l'option religieuse de Montesquieu est indissociable de l'entreprise d'observation et de compréhension rationnelles, où il déploie, comme l'on sait, une vaste sociologie des religions pour décrire leur action locale, leurs effets plus ou moins civilisateurs, leur manière d'agir sur les coeurs là où les lois civiles sont sans force. En étudiant leur degré de cohérence avec les différents systèmes politiques et comment elles les servent ou les entravent, il note le lien entre les gouvernements modérés et la religion chrétienne, les gouvernements despotiques et la religion mahométane; entre la monarchie et le catholicisme, la république et le protestantisme; enfin entre les rites, les croyances et les climats ( $E L$, XXIV et XXV). Comme dans les Lettres persanes, ce travail n'est pas qu'interrogatif ou descriptif, l'explication rationnelle des pratiques religieuses faisant apparaître d'elle-même un horizon prescriptif de tolérance et de modération. La comparaison des diverses manifestations de la religion est en soi porteuse d'une valeur universelle qui rejoint, à travers des justifications morales et laïques, les valeurs de douceur et de tolérance associées au départ avec la religion chrétienne. Alors que dans les Lettres persanes la relativisation des croyances révélait, comme en négatif, un noyau commun des religions, la sociologie « objective » de L'Esprit des lois devient l'éloge d'une justice humaine universelle que résument les "lois naturelles" mises en place au début de l'ouvrage, et énoncées sans référence à la religion: ces "rapports d'équité naturelle antérieurs à la loi positive qui les établit», incluent, outre le désir de se conserver, de se reproduire et de sociabiliser, l'instinct religieux lui-même (EL, I, 2).

On peut comprendre, à partir de là, la position subtile de Montesquieu sur les rapports de la religion et du pouvoir politique, qui illustre bien sa méthode d'ensemble. L'origine 
de la religion réside dans le besoin qu'en ont les sociétés, pense Montesquieu, puisque c'est avec la société qu'apparaît l'état de guerre entre les hommes, et que l'exigence de paix fait naître les lois, la morale et la religion ( $E L, I, 1-2)$. Mais il ajoute que la religion existait à l'état latent avant la formation des sociétés, qu'elle n'est donc pas créée de toutes pièces mais organisée par les politiciens pour leurs fins sur la base de dispositions naturelles et selon les conditions géographiques ( $E L$, XXIV, 24-26). Les gouvernants ont forgé une institution permettant de fortifier l'autorité des lois à partir d'instincts religieux fondamentaux: l'obéissance des hommes à ce qui leur est supérieur et l'amour vis-à-vis des autres hommes. Dans ce cadre, Montesquieu s'oppose à une utilisation politique directe de la religion, c'est-à-dire à sa réglementation par l'État, même $s^{\prime}$ il reconnaît la puissance qui résulte de cette unité $(E L, X X I V, 1)^{7}$. Si l'ordre politique requiert bien les sentiments de respect, de crainte, de fraternité que la religion entretient dans le coeur des hommes, c'est pourtant la séparation de la religion et de l'État et la tolérance des différents cultes, qui sont conformes à la raison et à la nature: c'est en restant séparée de l'État, en résistant à ses abus et en créant ainsi un équilibre, que la religion joue au mieux son rôle social. Cela dit, le principe général de la tolérance, aussi important soitil, est lui-même problématique, pour Montesquieu, car une tolérance universelle équivaut finalement à l'indifférence et donc à l'irreligion. Elle peut ouvrir la voie à des bouleversements religieux qui brusquent les esprits, affaiblissent des institutions qui tirent leur force de leur immutabilité; ce qui peut donner lieu à des affrontements si les différentes confessions deviennent trop zélées - comme Montesquieu le voit en France depuis la Réforme ( $E L, X X I V, 5)$, en particulier dans sa région bordelaise. D'où la solution prudentielle de Montesquieu, aussi méfiante à l'égard de l'intolérance de la religion d'État qu'à l'égard d'une ouverture généralisée à tous les possibles; une solution nourrie autant par la sagesse pratique que par l'exigence normative: "Ce sera une très bonne loi civile, lorsque l'Etat est satisfait de la religion déjà établie, de ne point souffrir l'établissement d'une autre. Voici donc le principe fondamental des lois politiques en matière de religion: quand on est maitre de recevoir dans un État une nouvelle religion, ou de ne pas la recevoir, il ne faut pas l'y établir; quand elle y est établie, il faut la tolérer » $(E L, X X V, 10)$. 
Cette même approche est à l'oeuvre pour tous les thèmes privilégiés de Montesquieu dans les deux ouvrages. Dans le cas de l'esclavage domestique, les Lettres accomplissent leur travail de relativisation par l'examen des degrés de subordination des femmes selon les pays ${ }^{8}$ et selon les religions ( $L P, C X L I$, CXVI); mais l'intrigue parallèle qui se déroule dans le sérail $\mathrm{d}^{\prime}$ Usbek fait transparaître une dénonciation virulente des arguments en faveur de la soumission naturelle des femmes et de la cruauté à leur égard ${ }^{9}$. Si le dénouement de cette intrigue est tragique, l'angoisse, la jalousie et l'échec d'Usbek étant sanctionnés par le suicide de Roxane, sa préférée ( $L P, C L X I)$, la souffrance psychologique et les contradictions politiques des personnages n'ouvrent pas cependant sur une éthique de la résignation, car le mal n'est pas renvoyé à un principe métaphysique régissant le monde. Ce mal est décrit plutôt de manière à mettre en évidence l'aveuglement et l'excès, et donc les tâches de la raison: celle-ci peut éviter aux hommes les erreurs dues à l'orgueil ou à l'ambition démesurée du pouvoir. Comme pour la religion, L'Esprit des lois reprend ce thème de manière plus explicative, en soulignant les raisons géographiques et climatiques de la servitude des femmes, et en démontrant l'inutilité de la polygamie. Celle-ci ne contredit pas seulement la nature des choses par ses conséquences nocives sur la vie sociale (par exemple, elle n'encourage pas la propagation de l'espèce, ce qui, on le sait, est une préoccupation majeure de Montesquieu); mais elle heurte aussi la nature éthique de l'homme en affaiblissant l'amour des hommes pour les femmes, sans apaiser l'esprit de jouissance masculin et sans relâcher les liens d'asservissement des femmes ( $E L, X V I, 6)$. Même approche dans le cas du droit familial, où Montesquieu montre que le sentiment d'un devoir éthique (l'obligation alimentaire des parents vis-à-vis des enfants) vient se superposer à l'instinct naturel de procréation sur lequel se fonde le mariage ${ }^{10}$. Enfin, dans la dénonciation du despotisme, de l'esclavage ou de la violence coloniale ( $E L$, III, 9, et XV; et $L P, C I V, C X V I I, C X X I)$, Montesquieu $\mathrm{s}^{\prime}$ attache à démonter les justifications contradictoires qu'on leur donne, et à révéler l'échec politique de ces options, de sorte que les hommes aperçoivent en quoi la limitation de leurs passions peut servir leur propre intérêt, leurs entreprises devenant alors plus efficaces ou leur pouvoir plus durable. Mais il indique en même temps qu'en évitant les abus et en réglant avec douceur les rapports humains, c'est une finalité éthique qui est en jeu, celle du bonheur et de la sécurité des 
citoyens; celle de la dignité de l'homme comme condition de sa liberté.

À partir de ces quelques exemples, on voit s'esquisser la profonde parenté méthodologique des deux ouvrages de Montesquieu. Dans les Lettres persanes la reconstruction positive du monde social ne prend pas la forme d'un mouvement de pensée identifiable par un narrateur spécifique. Plutôt la diversité des points de vue suggère sans cesse l'existence d'un tel narrateur, comme celui qui met en oeuvre cette diversité. En indiquant la raison de cette diversité, ce narrateur absent contribue à la reconstruction indirecte d'une autre vision des choses, et ainsi $\mathrm{du}$ relativisme se dégage une universalité qui a la forme d'une exigence de la raison, d'un effort vers l'universel à travers les préjugés. Là réside la force démonstratrice des personnages que sont Usbek et Rica: ouverts au rationalisme de l'Occident, ils ne cessent dans leurs lettres de faire alterner raison et superstition car ils n'en restent pas moins enracinés dans le despotisme oriental. Mais différemment l'un de l'autre: plus jeune et plus ingénu qu'Usbek, Rica est plus souple face à ses découvertes européennes, et à la fin il ne souhaite pas retourner en Perse; Usbek qui se veut en quete de sagesse, plus franchement ouvert à la différence et plus sensible à la modération des moeurs européennes, demeure malgré tout un despote dogmatique et brutal dans son harem. À partir des conventions et des faux absolus de chaque société, l'exercice de la raison comme dialogue et critique des préjugés de chacun dessine un horizon de vérité une; une unité des points de vue qui demeure incertaine, problématique, mais qui ouvre la possibilité d'une idée de "justice éternelle " telle que l'indique la lettre la plus célèbre qui nie que la justice soit réductible aux conventions humaines et qui la définit comme un « rapport de convenance qui se trouve réellement entre deux choses" ( $L P$, LXXXIII). Cette convenance désigne un idéal d'ordre qui est conforme à un penchant naturel des hommes, qui les habite comme un "principe intérieur " et qui s'identifie finalement à la raison comme amour de l'ordre de la nature ${ }^{11}$. Tous les hommes contemplent une même vérité, sur le modèle des vérités mathématiques de Platon, et en matière de justice il s'agit des caractères universels de la vie en commun: respect des lois, amour des hommes, piété envers ses parents ( $L P$, XLVI). Toutefois dans la vie sociale cet absolu reste formel, car les choses changent dans l'espace et le temps, et les hommes, portés par leurs passions, s'éloignent de ces caractères naturels. 
Il y a un ordre des choses désirable et désiré par les hommes, mais le plus souvent manqué par eux à cause de leur hâte, de leur ignorance, de leurs appétits excessifs. L'effort rationnel des hommes pour retrouver cet ordre naturel se traduit par des lois, lesquelles, adaptées aux circonstances, manifestent la justice en un lieu et un temps particuliers. Rationalisme et relativisme s'allient donc, car pas plus la justice n'est-elle réductible aux conventions des hommes, pas plus ne sauraitelle prendre une forme concrète en dehors de ces conventions. L'ordre alternatif ainsi dessiné ne saurait s'incarner dans tel ou tel comportement: il reste un horizon qui, à la fois, rend possible la critique du présent et n'existe que par cette critique. Le lecteur des Lettres persanes est ainsi amené sans cesse à constituer lui-même cet horizon normatif, la forme de l'ouvrage venant illustrer et soutenir à tous moments l'un de ses thèmes les plus constants: l'éloge de l'ouverture aux sciences et aux lettres, de l'interaction croissante des hommes dans le monde moderne, et bien sûr des vertus du commerce qui adoucit les moeurs et qui ouvre l'esprit à l'universel par l'expérience des différences ${ }^{12}$.

La méthode énigmatique et tant discutée de $L^{\prime}$ Esprit des lois retrouve cette idée d'un principe universel comme horizon d'une observation des variations des phénomènes, plutôt que celle d'un principe a priori commandant aux faits, ou celle d'un simple relativisme sans ordre possible. Pour le monde physique, Montesquieu adhère à une conception mécaniste: les lois mécaniques sont des rapports exprimant la constance des phénomènes, qui elle-même manifeste la puissance et la sagesse de Dieu ( $E L, I, 1)$. Mais pour le monde humain, la réalité est plus complexe car la liberté des hommes vient modifier leurs déterminations naturelles. Capable d'erreur et doué de liberté, l'homme est un être double, à la fois nature et esprit, ce qui rend les lois humaines plus ambivalentes. Elles n'en ont pas moins leur raison en ce qu'elles sont des rapports (à la géographie, à l'histoire, et entre elles), et il est possible de comprendre la totalité de ces rapports. C'est précisément cette totalité explicative que Montesquieu nomme "l'esprit des lois » et qui lui permet d'explorer l'articulation entre l'universel d'une raison primitive et le particulier des lois positives ( $E L$, I, 3) ${ }^{13}$. Rendre compte rationnellement de la diversité de ces lois, ce n'est pas la réduire à une unité principielle a priori, $c^{\prime}$ est rendre intelligible de quelle manière les hommes s'éloignent de leur constitution naturelle par l'erreur et la liberté; et $c^{\prime}$ est 
aussi les rappeler à leurs limites. Tout comme la religion les rappelle à leur Créateur, et la morale à eux-mêmes ( $E L, \mathrm{I}, 1$, dernier paragraphe), les lois positives les rappellent à leur sociabilité fondamentale ${ }^{14}$. L'extrême concision de Montesquieu quant au contenu de ce «droit naturel", nous semble confirmer cette fonction d'horizon méthodologique qu'il lui assigne ${ }^{15}$, car il s'empresse de dépasser ces fondements anthropologiques généraux vers le programme scientifique qu'ils rendent maintenant possible: comment les hommes s'éloignent du droit naturel ou comment ils le rejoignent, et quels sont les degrés d'effectivité de leurs essais tels que manifestés par les lois positives. On comprend ainsi que cette étude nécessite une constante ambivalence du descriptif et du normatif, sur laquelle, d'ailleurs, Montesquieu est à plusieurs reprises explicite ${ }^{16}$, et qu'elle s'appuie sur une conception de la liberté qui n'est fondée ni sur la conformité au droit naturel, ni sur la licence, mais sur la légalité. La loi pose une limite au pouvoir des hommes les uns sur les autres, en agissant comme contre-pouvoir. D'où la véritable liberté politique définie comme l'obéissance à des règles qui manifestent, par leur adéquation aux circonstances, la sociabilité naturelle des hommes ( $E L, X I, 3)$.

C'est une liberté ainsi définie qui est l'enjeu de la célèbre théorie des gouvernements; non pas en ce qu'un régime particulier la réaliserait, mais en ce que certains régimes permettent de la garantir par des lois. On comprend alors le sens et l'importance, pas toujours assez reconnue, de l'esprit de modération dans la typologie de Montesquieu. Plus que tripartite (république, monarchie, despotisme), sa classification est bipartite: d'un côté les régimes modérés où le pouvoir s'exerce de manière réglée, de l'autre le despotisme où le pouvoir s'exerce sans limite. Ce principe de modération est introduit discrètement (comme il se doit) par Montesquieu, quand il décrit les régimes démocratique et aristocratique animés l'un et l'autre par le "principe » républicain de la vertu: celle-ci est moins nécessaire, dit-il, en régime aristocratique où les lois des nobles encadrent le peuple et suscitent son obéissance volontaire, de façon que l'esprit de modération tend à remplacer celui de l'égalité qui requérait impérativement la vertu $(E L, V, 14)$. En fait la modération apparaît comme une catégorie de plus en plus opératoire dans L'Esprit des lois, devenant finalement celle par laquelle se définit l'État politique en tant que tel: celui où le pouvoir du gouvernement est modéré par la structure juridique, ce qui lui confère sa légitimité et son efficacité ( $E L$, VIII, 8) ${ }^{17}$. Ce principe ne s'illustre 
pas seulement par la condamnation du despotisme, mais aussi dans la discussion de la corruption des régimes, et particulièrement du régime démocratique. Celui-ci dégénère soit en perdant l'esprit d'égalité qui l'anime, soit en instaurant l'« égalité extrême » (EL, VIII, 2-3), laquelle manifeste, de la part du peuple cette fois, un exercice excessif du pouvoir ("délibérer pour le sénat, exécuter pour les magistrats et dépouiller tous les juges») qui le fait devenir un tyran pour lui-même. La critique de Montesquieu ne consiste pas ici à renvoyer cet excès à une égalité naturelle des hommes, car l'égalité extrême qu'il combat est justement l'amour de l'égalité anarchique de l'état de nature et non la seule véritable égalité qui est conventionnelle et réglée par des lois. Seules celles-ci peuvent modérer les élans du peuple et lui permettre d'exercer son pouvoir dans l'exacte mesure où il consent à le limiter. Il est vrai que le peuple étant, selon Montesquieu, inapte par nature à gérer les affaires publiques, ce régime ne saurait mettre en place l'équilibre requis pour assurer le bonheur des hommes, comme le pourrait la monarchie constitutionnelle qui a la faveur de Montesquieu. Il demeure que, dans le cadre de son "principe", de sa logique propre, ce régime ne saurait se maintenir qu'en modérant sa passion égalitaire.

Enfin, on aperçoit ainsi la portée méthodologique du célèbre "système » de la libertée ${ }^{18}$, décrit dans L'Esprit des lois par référence à la Constitution anglaise ( $E L, \mathrm{XI}, 6)$. Il ne s'agit pas de fournir la recette institutionnelle qui incarnerait la liberté politique, car aucun gouvernement, aussi modéré soit-il dans ses principes, n'est à l'abri de la dégénérescence. En décrivant comment le " pouvoir arrête le pouvoir », Montesquieu dessine un système tendu vers un idéal de liberté politique, où l'équilibre des forces vise à neutraliser les velléités despotiques toujours à l'oeuvre. Tous les régimes étant susceptibles de se corrompre et tous étant imparfaits, Montesquieu délimite d'abord ce qui est acceptable et fondé rationnellement en dénonçant le despotisme; puis il cherche quelle combinaison d'imperfections peut fournir le plus de garanties pour assurer la liberté politique. Mais il $n^{\prime}$ espère pas pour autant atteindre le bon régime, car c'est cette liberté des hommes elle-même qui interdit d'en finir avec l'incertitude et le travail d'équilibrage des forces. La valeur de liberté se formule donc négativement ici, puisqu'on cherche seulement à empêcher que le pouvoir s'exerce sans limite et sans partage. Cette valeur visée par la légalité et la modération est, à la fois, universelle et susceptible de recevoir des contenus changeants 
selon l'état des forces en présence. Les instruments de cette politique sont la fragmentation du pouvoir et l'équilibre des pouvoirs, mais selon des formules nécessairement différentes dans chaque société et dans l'histoire.

Cette démarche d'ensemble nous semble contenir au moins deux enseignements importants; et, quoique très liés, nous les distinguerons ici dans l'espoir de mieux préciser la place de Montesquieu dans les débats de la pensée politique.

L'originalité de son oeuvre réside d'abord dans la manière dont elle fait $s^{\prime}$ articuler l'universalisme et le relativisme. C'est précisément cette ambivalence que lui reproche une tradition importante de la pensée qui, simultanément, prétend s'inspirer de sa méthode. Nous pensons ici au positivisme de Comte pour lequel la science sociale mise en oeuvre dans L'Esprit des lois pour étudier les conditions particulières des sociétés (surtout à partir des climats, ce qui est, pour Comte, la contribution la plus importante de l'ouvrage car la seule qui soit pleinement scientifique), est transformée en un nouvel absolu ${ }^{19}$. Les conditions géographiques dont le législateur, selon Montesquieu, doit tenir compte pour faire de bonnes lois, deviennent chez Comte le fondement d'une universalité qui doit régir la politique: celle de l'observation des faits et de leur enchaînement causal. Si Comte se félicite de ce que Montesquieu se distingue par là des utopistes de son époque, au premier titre de ce qui deviendra la "politique métaphysique " de Rousseau, il déplore néanmoins qu'il n'ait pas été jusqu'au bout de son intuition et qu'il ne se soit pas débarrassé de toute métaphysique. Comte vise ici avant tout le premier Livre de L'Esprit des lois, l'horizon prescriptif qui y est mis en place comme "équité naturelle " et qui interdit dans la suite de l'ouvrage toute absorption de l'éthique par la science. On trouve le même reproche de la part de Durkheim, pour lequel cette ambivalence entache la révolution méthodologique amorcée par l'ouvrage ${ }^{20}$. Au lieu de s'en tenir à la description déterministe du monde social, Montesquieu fait des hypothèses basées sur un droit naturel; et, supposant les hommes intelligents, libres, sujets à l'erreur, supposant les lois du monde humain moins bien respectées que celles du monde physique, Montesquieu fait intervenir une contingence dans la causalité naturelle. Bref, dit Durkheim, il fait un "mélange de science et d'art " et son oeuvre appelle de ce fait une épuration pour atteindre le statut d'une authentique 
science du social. Ajoutons enfin que l'interprétation plus récente et fort connue d'Althusser est dans le même esprit, même si elle ne décèle pas chez Montesquieu ces relents de métaphysique et se félicite, à tort nous semble-t-il, qu'il se soit débarrassé de toute référence prescriptive à des normes pour s'en tenir aux faits et aux lois ${ }^{21}$.

En regard de cette tradition interprétative pour laquelle l'héritage essentiel de Montesquieu est, ou devrait être, la soumission de l'éthique à la science, il nous semble qu'il faut placer Montesquieu fermement aux côtés de Rousseau. Le rapprochement avec Rousseau sur cette question peut étonner quand on se rappelle son célèbre jugement sur Montesquieu, selon lequel ce dernier ne traiterait que du droit positif et non des "principes du droit politique " c'est-à-dire de ce qui doit être ${ }^{22}$. Mais nous pensons avoir montré justement que Rousseau se méprend en réduisant ainsi la portée de L'Esprit des lois; et que quelles que soient les divergences entre leurs deux pensées quant à la politique (nous allons y venir), elles ont en commun la référence à un horizon d'universalité éthique. Si Montesquieu est un penseur résolument moderne par sa conception «raisonnée » du droit naturel, il n'est nullement relativiste: il est attaché à un étalon normatif qui reste à l'horizon de ses observations et qui donne sens à son idée de légalité-modération. C'est d'ailleurs ce qu'ont bien vu ses meilleurs interprètes récents, qui montrent comment se combinent et se chevauchent chez lui la description scientifique et la prescription éthique, la quête neutre de connaissance et le jugement selon le bien public ${ }^{23}$. Montesquieu dit bien qu'il s'agit pour lui non d'évaluer les divers usages mais d'en rendre raison, de dire "ce qui est et non pas ce qui doit être " ( $E L, I V, 2$, note); toutefois il ne s'en tient pas là, ses conseils au législateur visant la modification des effets de la causalité naturelle en vue $d^{\prime} u n$ ordre éthique. C'est bien selon une valeur que sont jugés le despotisme, l'Inquisition, les conquêtes coloniales ou même "l'égalité extrême ", cette valeur définissant la fin du droit et donc la tâche du législateur: savoir la traduire en règle en agissant si nécessaire sur les effets du déterminisme naturel. Cette valeur est inscrite dans la conscience même de l'homme, comme chez Rousseau, mais si elle a une origine subjective et dépend donc de la volonté, elle renvoie aussi à un ordre qui transcende la subjectivité de chacun, un ordre que l'esprit du législateur peut discerner à travers les conditions particulières et que son œuvre doit 
trouver les moyens de rejoindre. Cette universalité est « de parcours" ou "de méthode " ${ }^{24}$, rendant possible la confrontation des différences, fournissant une mesure et une direction pour les tâches à accomplir; mais sans ériger en absolu un usage particulier et sans renoncer non plus à tout jugement transculturel.

La caractérisation du statut théorique de Montesquieu à travers cet horizon prescriptif doit être complétée d'une autre, laquelle, en apparence, va en sens inverse, mais qui chez Montesquieu s'articule au problème dont nous venons de parler. En effet, on a vu que la légalité-modération n'est pas une règle qui s'incarne dans un type unique de régime politique: ce n'est qu'enracinée dans la spécificité géographique et historique de chaque nation que l'oeuvre du législateur peut être effective, et sa justice, aussi généreuse soit-elle, ne pas être une abstraction. C'est à cette exigence de pluralisme dans l'action du législateur que répond le concept $\mathrm{d}^{\prime}$ " esprit général " d'une nation ( $E L$, XIX, 4). S'il convient, selon Montesquieu, d'être attentif à la simplicité et à la quantité de lois à établir dans une nation ( $E L, X X I X, 1$ et 16$)$, et de bien distinguer entre les types de lois selon leur objet propre $(E L, X X V I)$, il faut surtout savoir respecter le caractère pluriel de l'oeuvre législatrice en ne séparant pas les lois des circonstances dans lesquelles elles ont été faites et généralement en se défiant de l'uniformité (EL, XXIX, 14 et 18 ).

Comme pour le "mélange de science et d'art " ci-dessus, cet enjeu de l'uniformité des lois a fait l'objet d'une critique fondamentale de la pensée de Montesquieu, celle de Condorcet ${ }^{25}$, qui permet de mettre en évidence l'originalité de la méthode décrite plus haut. Comme Montesquieu, Condorcet entend substituer le travail de la raison au droit naturel de la métaphysique ancienne ou chrétienne; mais de cette raison procèdent chez lui non seulement des principes de justice universels, mais également des lois qui sont aussi universelles que l'était le droit naturel de la tradition. Les lois découlent directement des principes de justice et sont valables partout, en tout temps et pour tous les hommes - comme une proposition mathématique, dit-il, est vraie pour tous ${ }^{26}$. Le travail du législateur s'apparente ainsi à celui du scientifique, ce qui permet à Condorcet d'envisager, non seulement un système unique de lois, mais un État unique dépassant les divisions nationales, et une langue unique oeuvrant à l'unité du genre humain grâce aux lumières de la raison ${ }^{27}$. Si Montesquieu n'appartient pas moins que Condorcet à cet 
esprit des Lumières, on a vu que pour lui l'oeuvre du législateur ne saurait être commandée seulement par cet universalisme, car les lois reçoivent leur légitimité et leur sens, à la fois, $d^{\prime}$ un horizon de justice universel, et des conditions particulières où ces lois s'inscrivent et d'où elles émergent. Les conditions géographiques et historiques déterminent des croyances et des moeurs auxquelles les lois doivent répondre, soit en $s^{\prime} y$ ajustant, soit en corrigeant leurs effets néfastes. Pour Condorcet, la "grandeur du génie d'un pays", que Montesquieu incite le législateur à comprendre et à respecter ( $E L$, XIX, 5), est une de ces notions vagues qui "frappent les petits esprits et plaisent aux hommes corrompus ${ }^{28}$, car cela ne désigne finalement rien d'autre que les préjugés. Or s'il faut bien des règlements particuliers selon les habitudes ou les comportements privés dans chaque nation, en matière de lois une mesure invariable s'impose, car la fonction des lois, qui s'occupent de la vie publique, est précisément de combattre les préjugés en dépassant les enracinements particuliers et les opinions des groupes. Il n'est donc pas concevable, pour Condorcet, que deux lois différentes soient toutes deux justes ou également utiles.

Ce sont deux conceptions de la politique qu'on voit se dessiner ici: une pensée univoque où la politique est régie par une science universelle renversant les préjugés au nom d'une unité fondamentale du genre humain; et une pensée duelle ou plurielle où l'exigence d'universalité est renvoyée aux conditions particulières, et où ces conditions sont ellesmêmes renvoyées à un horizon universel, dans un va et vient qui définit les tâches propres du législateur. Par cette dernière méthode, Montesquieu se rattache à une longue tradition héritière d'Aristote, et par laquelle, à l'époque moderne, il s'oppose cette fois à la démarche de Rousseau. Il faut certes prendre garde de ne pas simplifier la politique rousseauiste en négligeant l'héritage de Montesquieu qu'elle intègre de manière évidente, et la souplesse que manifeste cette politique particulièrement dans ses textes moins connus ${ }^{29}$. Il reste que l'impulsion fondamentale du Contrat social est celle d'une quête de légitimité réalisable par un modèle d'inspiration platonicienne. La politique de Rousseau est au fond une anthropologie morale appliquée à la cité, où le législateur agit selon sa connaissance du bien; alors que Montesquieu reconnaît une certaine indétermination quant à ce qu'est le bien politique, ce qui interdit dès lors tout schéma unique de législation qui prétendrait répondre à l'universalité de la condition humaine. A cet égard, l'approche 
par Montesquieu de la place de la religion dans la cité, telle qu'évoquée ici plus haut, est significative. Alors que Rousseau (comme Hobbes) affirme la fonction politique de la religion (garante de la paix civile dans le cadre de l'absolutisme, pour l'Anglais; garante de la santé morale dans le cadre de la souveraineté populaire, pour le Genevois) et qu'il l'intègre au pouvoir comme "religion civile", Montesquieu (comme Locke, et plus tard Tocqueville) donne bien à la religion un rôle de moralisation de la société mais sans l'absorber dans le pouvoir politique. Cette politique plurielle signifie que s'il y a, pour Montesquieu, un mal politique clairement identifiable ( $c$ 'est le despotisme), il y a cependant plusieurs biens politiques possibles, dont seul l'horizon de modération peut tenir compte sans pour cela s'abandonner au relativisme des usages particuliers ${ }^{30}$. Le domaine des lois humaines est celui du bien, non celui du " meilleur " ou du " parfait " qui est le domaine inchangeable des lois divines ( $E L, X X V I, 2)$. La politique doit donc tenir compte non seulement des variations dans le temps et l'espace mais de l'imperfection inhérente à tous les régimes existant réellement. La politique de Rousseau, elle, n'a finalement qu'un critère de légitimité: la liberté des citoyens associés, réalisée par la souveraineté du peuple. Et dès lors que le bien politique est connu et déterminé comme un, et que ce bien est traduit en pouvoir légitime, limiter ce pouvoir c'est s'attaquer à la légitimité. Dans ce cadre théorique, il n'y a pas plus de justification à vouloir limiter l'action du philosophe-roi de Platon qu'il n'y en a à chercher à " diviser » ou à « aliéner » la souveraineté de Rousseau. Ce dernier, comme nous l'avons dit, n'est nullement aveugle à la diversité des situations et le Contrat social reconnaît explicitement sa dette envers Montesquieu à cet égard ${ }^{31}$, mais cet enjeu reste au fond extérieur au problème politique luimême. Chez Montesquieu en revanche, la diversité est constitutive de la politique; mieux, le problème politique consiste précisément en cette prise en charge du divers.

Peut-on dire que Montesquieu se révèle, de cette manière, singulièrement plus moderne que Rousseau, ce dernier étant davantage attaché au modèle antique de la cité vertueuse où politique et morale ne font qu'un? Le sens qu'ils donnent respectivement au mot "vertu " le suggèrerait: sens seulement politique, requis pour assurer la sécurité individuelle et sanctionnant donc une séparation du public et du privé, chez Montesquieu ( $E L, I V, 4-6)$; sens moral, désignant une qualité humaine que la vie politique requiert et réalise à la fois, chez Rousseau. 
Mais ne faut-il pas plutôt distinguer entre les Anciens pour bien statuer à cet égard?

Ainsi si Platon et Aristote définissent l'un et l'autre la cité par référence à sa fin, leurs conceptions de cette cité sont, comme l'on sait, bien différentes. Platon privilégie son degré d'unité interne, à l'image de celle de la famille, alors qu'Aristote critique ce critère d'unité et distingue famille et cité, cette dernière se caractérisant justement par sa pluralité interne. Cette opposition sur l'homogénéité interne se retrouve sur le plan de ce qu'on pourrait appeler l'épistémologie politique. Dans La République le bon régime est unique, et ce sont les circonstances concrètes qui produisent des formes autres et dégradées en regard de ce modèle qui n'est pas réalisé ${ }^{32}$. Chez Aristote, par contre, la question du meilleur régime n'est jamais nettement résolue par une description unique, et il reste toujours une dimension d'indétermination à la base de cette politique ${ }^{33}$. Celle-ci $n^{\prime}$ en est pas moins normative, la recherche d'une constitution excellente ne se dément jamais et s'appuie sur des critères applicables universellement: que le gouvernement agisse en vue de l'avantage commun et non de celui des gouvernants eux-mêmes; et que chacun dans la cité reçoive en proportion de son excellence. Mais cette politique de l'excellence est une politique plurielle, car la constitution n'est excellente (durable et efficace) que selon l'état du groupe qu'elle doit régir. Il y a donc une diversité de l'excellence qui fait appel à la sagesse pratique du législateur, à sa prudence, à son sens de l'opportunité et du possible. Plusieurs régimes sont acceptables par conséquent, sans pourtant que soit abandonné tout critère universel. Il s'agit bien $d^{\prime}$ assurer par une bonne constitution la réalisation de bonnes lois, suscitant des habitudes vertueuses chez les citoyens et leur permettant d'atteindre le bonheur; mais l'excellence ne saurait avoir un seul visage, l'enjeu propre du politique étant de discerner et de mettre en oeuvre celui qui convient. Cela dit, la normativité chez Montesquieu n'est pas celle du droit naturel des Anciens. La politique chez lui est d'abord appelée à garantir la sécurité et la tranquillité des individus, et le rapprochement avec le pluralisme $d^{\prime}$ Aristote ne doit pas faire oublier ce clivage ${ }^{34}$. Il met en évidence néanmoins les racines de ce second enseignement, pluraliste, que nous voulions dégager de sa méthode d'ensemble.

Notons pour conclure que l'importance de ces deux thèmes, universalisme et pluralisme, reçoit une confirmation éloquente 
dans le texte même de Montesquieu. Le Livre XXIX de L'Esprit des lois, mentionné plus haut par rapport à Condorcet, ne fait pas que combattre, dans ses demiers chapitres, l'esprit d'uniformité chez le législateur. Il s'ouvre par un chapitre sans équivoque, et souvent cité, sur l'esprit de modération; et Montesquieu nous semble avoir voulu ainsi afficher et lier, en conclusion à tout son ouvrage ${ }^{35}$, ses deux thèses fondamentales. L'universel de Montesquieu se définit comme légalité-modération et se réalise institutionnellement par l'équilibre et la limitation des pouvoirs. Contre l'homogénéisation et l'intégration des forces sociales en une unité collective, Montesquieu défend le caractère salutaire d'un certain conflit social indépassable: la concurrence dans le commerce, la compétition des corps intermédiaires ou des sectes religieuses, les empêchements que s'infligent les deux chambres et le Roi en Angleterre... Tout cela représente des jeux de pouvoirs réglés qui garantissent la liberté en équilibrant et en limitant ces pouvoirs. Ce pluralisme interne à la société en vient à réaliser la modération, les deux termes définissant ensemble l'horizon du législateur. On peut alors se demander si, pour Montesquieu, ce pluralisme et cette modération sont eux-mêmes susceptibles d'une politique unitaire. L'Esprit des lois nous semble l'interdire, en n'envisageant nullement qu'un système de lois puisse fixer définitivement les équilibres requis (comme on l'a vu pour la Constitution d'Angleterre). Montesquieu dit bien que les lois garantissent la sécurité des citoyens dans la mesure où elles assurent la stabilité de l'État, donc dans la mesure où elles perdurent; mais elles ne doivent pas moins être sujettes au changement, au conflit de l'ancien et du nouveau, pour que la liberté des hommes, qui à la fois insuffle sa vie à l'ordre politique et constitue sa finalité, ne soit pas brimée $(E L, X X V, 2)$. C'est cette fixité relative, cet alliage de mobilité et de stabilité, que ne cesse de rechercher L'Esprit des lois, en une circularité toujours reprise où les lois garantissent le pluralisme et le conflit réglé, en même temps qu'elles se nourrissent d'eux pour se renouveler.

Il y a une irréductible diversité, propre à la réalité politique, qu'on méconnaît en érigeant en absolu un modèle particulier de vie collective, aussi généreux soit-il, dans l'espoir de ramener ce divers à l'unité au nom de la morale ou de la connaissance philosophique. Mais cette autonomie de la politique n'est pas moins pervertie si l'on abandonne en son nom toute référence dépassant le droit positif et permettant de le juger. Montesquieu nous semble admettre en même temps ces deux exigences et 
chercher, par la modération, la formule de leur équilibre la formule, pourrait-on dire, d'une "Idée régulatrice " en matière politique. $S^{\prime}$ il est le penseur du pluralisme politique, et l'un des meilleurs garants, contre le moralisme rousseauiste, de la tradition aristotélicienne d'autonomie du politique à l'époque moderne, il est aussi, à l'instar de Rousseau, l'un des penseurs qui articule le plus savamment l'universel et le particulier en politique, contre le scientisme qui détourne au XIXe siècle la pensée humaniste des Lumières, et contre un certain relativisme que nous connaissons bien au XXe siècle. La portée de son oeuvre aujourd'hui ne se livre ainsi que si l'on évite, au départ, de l'enfermer dans des clivages ou des réputations rigides, et si l'on réussit à dépasser le malaise initial que provoque une oeuvre où se superposent les points de vue du fait et de la valeur ${ }^{36}$, où se croisent les langages de l'être et du devoir-être, la référence au droit naturel et la modernité de la raison scientifique; une oeuvre où sont pensées ensemble les exigences politiques de la stabilité et du renouvellement, de la pluralité et de la cohésion sociale.

Faculté de philosophie, Université Laval.

\section{NOTES}

1. Voir, par exemple, malgré leurs approches fort différentes, les ouvrages de A. Finkielkraut, La Défaite de la pensée, Gallimard, Paris, 1987; T. Todorov, Nous et les autres. La réflexion française sur la diversité humaine, Seuil, Paris, 1989; L. Ferry, Philosophie politique I et II, P.U.F., Paris, 1984; P. Caussat, De l'identité culturelle, Desclée de Brouwer, Paris, 1989.

2. Voir A. Bloom, L'Âme désarmée, Julliard/Guérin littérature, Montréal, 1987; et surtout les écrits de son inspirateur, L. Strauss, par exemple: Droit naturel et histoire, Plon, Paris, 1954. Et pour l'antihumanisme, rappelons la critique de certains de ses représentants français par L. Ferry et A. Renaut, La Pensée 68, Gallimard, Paris, 1985.

3. Voir, par exemple, la brève mais significative référence aux " principes universels d'équité » de Montesquieu, chez Finkielkraut, ouvrage cité, p. 14; et le long développement laudatif chez Todorov, ouvrage cité, ch. 5 .

4. Les références à ces deux oeuvres seront données entre parenthèses comme suit: ( $L P$ suivi du numéro de la lettre), pour les Lettres persanes, dans CEuvres complètes, Bibliothèque de la Pléiade, Paris, 
1949-51, Tome I. Et: (EL suivi du numéro du Livre et du chapitre), pour De l'Esprit des lois, dans O. C., Tome II.

5. Voir, par exemple, les lettres sur la bulle Unigenitus contre les jansénistes ( $L P, X X I V)$, sur l'Inquisition en Espagne et au Portugal (XXIX), ou sur la révocation de l'Édit de Nantes par Louis XIV (LXXXV).

6. Au nom de l'utilité sociale du christianisme, Montesquieu rejette vigoureusement les célèbres arguments de Bayle sur les maux produits par les abus de cette religion ( $E L, X X I V, 2$ et 6 ), même s'il ne cache pas son estime pour ce "grand homme".

7. Dans les Considérations sur les causes de la grandeur des Romains et de leur décadence, Montesquieu souligne que la "religion patriotique " est l'un des fondements de la puissance des Romains; mais il note aussi les effets nocifs de la non-séparation de l'Église et de l'État dans l'Empire romain oriental, en suggérant au lecteur d'apercevoir ces mêmes effets en France. Pas de dénonciation féroce à la Voltaire chez Montesquieu, mais une incitation à réfléchir sur les conditions nécessaires de la séparation naturelle de l'Église et de l'État. La voie suggérée est celle d'une renaissance des lettres, dont les Considérations (comme les Lettres persanes) sont déjà elles-mêmes une composante. (Voir dans O. C., Tome I, surtout ch. X et XXII).

8. De la subordination totale en Perse ( $L P, L X I I)$, à celle, moindre, de l'Italie (XXIII), jusqu'à Paris où les femmes "gouvernent " (CVII), en passant par la Moscovie où elles aiment être battues (LI)!

9. Cette dénonciation est aussi argumentée explicitement, quoique brièvement, par un "philosophe galant " qui nie que la soumission des femmes soit fondée sur la loi naturelle (LP, XXXVIII).

10. Voir J. M. Trigeaud, "La liberté du législateur civil selon Montesquieu », dans La Pensée politique de Montesquieu, Cahiers de philosophie politique et juridique de l’Université de Caen, n 7, Vrin, Paris, 1985.

11. Comme souvent au XVIIIe siècle, cette idée de justice s'exprime à travers une certaine ambivalence, puisqu'il s'agit d'un ensemble immanent de relations, compréhensible en lui-même, mais qui correspond néanmoins à un principe transcendant qui détermine la nature des choses. Cela dit, même sans Dieu, assure Montesquieu, les hommes resteraient attachés à cette justice immanente: libres qu'ils seraient du joug de la religion, ils ne le seraient pas de celui de l'équité ( $L P$, LXXXIII).

12. Le personnage de Rhedi soutient la thèse de l'heureuse ignorance des peuples primitifs contre les caractères efféminés et les moeurs amollies de l'époque moderne (thèse à laquelle Rousseau donnera une forme si frappante quelques années plus tard dans son Discours sur les sciences et les arts). Usbek s'efforce de répondre à l'exigence d'homogénéité sociale par l'optimisme de l'ouverture et de la tolérance, aux réflexes de repli et d'appartenance par le pluralisme, c'est-à- 
dire qu'il prête sa voix à la démarche même de l'ouvrage dont lui et son interlocuteur font justement partie ( $L P, C V$ et $C V I$ ).

13. Todorov propose à ce sujet un schéma utile, où l'esprit des lois se présente au carrefour de quatre vecteurs: d'en haut il est nourri par le droit naturel et d'en bas par les lois positives; de la gauche par les types de gouvernements et de la droite par les causes physiques et morales qui forment l'esprit des nations: "Droit naturel et formes de gouvernement dans $L^{\prime}$ Esprit des lois », dans Esprit, $\mathrm{n}^{\circ} 3,1983$, p. 36 .

14. Parmi les lois naturelles énoncées par Montesquieu (voir ci-dessus), c'est la sociabilité qui est la plus importante, car c'est par elle qu'il se rattache à la tradition aristotélicienne et s'oppose radicalement à Hobbes ( $E L, \mathrm{I}, 2$; et la fameuse fable des Troglodytes: $L P, \mathrm{XI}$-XIV).

15. Montesquieu lui consacre huit paragraphes dans $L^{\prime}$ Esprit des lois (I, 2), et n'y revient plus explicitement.

16. Résumant, par exemple, son projet dans sa "Défense de l'Esprit des lois », Montesquieu écrit: "On peut dire que le sujet en est immense; qu'il embrasse toutes les institutions qui sont reçues parmi les hommes; puisque l'auteur distingue ces institutions; qu'il examine celles qui conviennent le plus à la société et à chaque société; qu'il en cherche l'origine, qu'il en découvre les causes physiques et morales; qu'il examine celles qui ont un degré de bonté par elles-mêmes et celles qui n'en ont aucun; que de deux pratiques pernicieuses il cherche celle qui l'est plus et celle qui l'est moins; qu'il y discute celles qui peuvent avoir de bons effets à un certain égard, et de mauvais dans un autre ». O. C., Tome II, p. 1137-8 (nous soulignons).

17. Voir S. Goyard-Fabre, La Philosophie du droit de Montesquieu, Klincksieck, Paris, 1973, ch. III.

18. Mes Pensées, 633, dans O. C., Tome I, p. 1153.

19. A. Comte, Cours de philosophie positive, Physique sociale, quaranteseptième leçon, Hermann, Paris, 1975.

20. E. Durkheim, Montesquieu et Rousseau précurseurs de la sociologie, M. Rivière, Paris, 1966.

21. L. Althusser, Montesquieu, la politique et l'histoire, P.U.F., Paris, 1959.

22. "Le seul moderne en état de créer cette grande et inutile science, écrit Rousseau en parlant du droit politique, eut été l'illustre Montesquieu. Mais il n'eut garde de traiter des principes du droit politique, il se contenta de traiter du droit positif des gouvernements établis, et rien au monde $n^{\prime}$ est plus différent que ces deux études [...]. Il faut savoir ce qui doit être pour bien juger de ce qui est ". Émile ou de l'éducation, dans Oeuvres complètes, Pléiade, Tome IV, pp. 8367. 
23. Voir en particulier: S. Goyard-Fabre, ouvrage cité; R. Aron, Les Étapes de la pensée sociologique, Gallimard, Paris, 1967; et plus récemment G. Benrekassa, Montesquieu, la liberté et l'histoire, Librairie générale française, Paris, 1987, qui se veut une réponse à Althusser.

24. Ce sont les termes utilisés par Todorov dans sa tentative de réhabiliter "l'humanisme tempéré » des Lumières contre ses plus farouches critiques au XXe siècle, en l'occurrence Lévi-Strauss: ouvrage cité, p. 94.

25. Condorcet, “Observations sur le XXIXe Livre de L'Esprit des lois ", dans Oeuvres, (éd. Arago), Firmin Didot Frères, 1847-9, Tome I. Nous utilisons le texte tel que reproduit dans les Cahiers de philosophie politique et juridique de l'Université de Caen, $\mathrm{n}^{\circ} 7$, ouvrage cité.

26. “Comme la vérité, écrit Condorcet, la raison, la justice, les droits des hommes, l'intérêt de la propriété, de la liberté, de la sûreté, sont les mêmes partout, on ne voit pas pourquoi toutes les provinces d'un État, ou même tous les États, n'auraient pas les mêmes lois criminelles, les mêmes lois civiles, les mêmes lois de commerce, etc. Une bonne loi doit être bonne pour tous les hommes, comme une proposition vraie est vraie pour tous. Les lois qui paraissent devoir être différentes suivant les différents pays, ou statuent sur des objets qu'il ne faut pas régler par des lois, comme sont la plupart des règlements de commerce, ou bien sont fondées sur des préjugés, des habitudes, qu'il faut déraciner; et un des meilleurs moyens de les détruire est de cesser de les soutenir par des lois ". Ibid., p. 152.

27. Condorcet, Esquisse d'un tableau historique des progrès de l'esprit humain, dans Oeuvres, Tome VI. La dixième "époque " du tableau développe l'idée d'une organisation scientifique de la société fondée, entre autres, sur le calcul des probabilités.

28. Condorcet, “Observations...», p. 153.

29. Sur les limites d'une opposition rigide entre la "convenance» chez Montesquieu et la "légitimité " chez Rousseau, voir la première partie de notre article: "La question de l'appartenance: Montesquieu, Rousseau et la Révolution française ", dans Revue canadienne de science politique, XXII, $\mathrm{n}^{\circ} 2,1989$.

30. Voir la remarquable analyse de B. Manin, "Montesquieu et la politique moderne », dans Montesquieu, Cahiers de philosophie politique, Université de Reims, n² 2-3, Ousia, Bruxelles, 1985.

31. Du Contrat social, Livre III, ch. 8.

32. S'il est vrai que dans Les Lois Platon infléchit sa position et consent à un régime moins parfait que celui de La République, c'est encore l'idée d'un modèle politique unique, caractérisé par l'unité interne, qui reste à l'oeuvre. D'autre part, il va de soi que le rapprochement esquissé ici entre Platon et Rousseau néglige tout ce qui sépare, par ailleurs, une politique fondée sur l'Idée du 
Bien, ouvrant sur la contemplation d'un autre monde, et une politique fondée sur l'idéal du juste, appelant à un effort pour le faire exister dans le réel.

33. L'introduction de la nouvelle traduction de La Politique d'Aristote, due à $P$. Pellegrin, rappelle fort judicieusement les arguments allant dans ce sens; voir Les Politiques, Garnier-Flammarion, Paris, 1990.

34. L'ouvrage de T. Pangle, Montesquieu's philosophy of liberalism, University of Chicago Press, 1973, privilégie à ce point ce clivage entre Anciens et Modernes (selon l'inspiration de Strauss), qu'il affirme la profonde parenté des positions de Hobbes et Montesquieu sur la liberté. Pour la critique de cet ouvrage, voir Manin, article cité.

35 . On peut considérer ce Livre XXIX comme la conclusion théorique de L'Esprit des lois: il n'est suivi que de deux Livres historiques sur les lois féodales chez les Francs, que Montesquieu a ajoutés, selon Shackleton, à la demande de son éditeur (Montesquieu. A Critical Biography, Oxford University Press, 1961, ch. X).

36. Voir S. Goyard-Fabre, "De la philosophie de Montesquieu et de son actualité ", dans Revue de métaphysique et de morale, $n^{\circ} 76,1971$, p. 322. 